\title{
Ketamine Treatment and Global Brain Connectivity in Major Depression
}

\author{
Chadi G Abdallah ${ }^{1,2}$, Lynnette A Averill, ${ }^{1,2}$, Katherine A Collins ${ }^{3}$, Paul Geha ${ }^{1,2}$, Jaclyn Schwartz ${ }^{4}$, \\ Christopher Averill, ${ }^{1,2}$, Kaitlin E DeWilde ${ }^{4}$, Edmund Wong, ${ }^{5}$, Alan Anticevic ${ }^{1,2,6,7}$, Cheuk Y Tang ${ }^{5}$, \\ Dan V losifescu ${ }^{3,4,8}$, Dennis S Charney ${ }^{3,4,8}$ and James W Murrough ${ }^{*, 3,4,8}$
}

'Clinical Neuroscience Division, VA National Center for PTSD, West Haven, CT, USA; ²Department of Psychiatry, Yale University School of Medicine, New Haven, CT, USA; ${ }^{3}$ Mood and Anxiety Disorders Program, Department of Psychiatry, Icahn School of Medicine at Mount Sinai, New York, NY, USA; ${ }^{4}$ Friedman Brain Institute, Icahn School of Medicine at Mount Sinai, New York, NY, USA; ${ }^{5}$ Department of Radiology, Icahn School of Medicine at Mount Sinai, New York, NY, USA; ${ }^{6}$ Department of Psychology, Yale University, New Haven, CT, USA; ${ }^{7}$ Interdepartmenal Neuroscience Program, Yale University School of Medicine, New Haven, CT, USA; ${ }^{8}$ Fishberg Department of Neuroscience, Icahn School of Medicine at Mount Sinai, New York, NY, USA

\begin{abstract}
Capitalizing on recent advances in resting-state functional connectivity magnetic resonance imaging (rs-fcMRI) and the distinctive paradigm of rapid mood normalization following ketamine treatment, the current study investigated intrinsic brain networks in major depressive disorder (MDD) during a depressive episode and following treatment with ketamine. Medication-free patients with MDD and healthy control subjects $(\mathrm{HC})$ completed baseline $r s-f c M R I$. MDD patients received a single infusion of ketamine and underwent repeated $r s-f c M R I$ at $24 \mathrm{~h}$ posttreatment. Global brain connectivity with global signal regression ( $\mathrm{GBCr}$ ) values were computed as the average of correlations of each voxel with all other gray matter voxels in the brain. MDD group showed reduced GBCr in the prefrontal cortex (PFC) but increased GBCr in the posterior cingulate, precuneus, lingual gyrus, and cerebellum. Ketamine significantly increased GBCr in the PFC and reduced $\mathrm{GBCr}$ in the cerebellum. At baseline, 2174 voxels of altered $\mathrm{GBC} r$ were identified, but only 310 voxels significantly differed relative to controls following treatment (corrected $\alpha<0.05$ ). Responders to ketamine showed increased GBCr in the lateral PFC, caudate, and insula. Follow-up seed-based analyses illustrated a pattern of dysconnectivity between the PFC/subcortex and the rest of the brain in MDD, which appeared to normalize postketamine. The extent of the functional dysconnectivity identified in MDD and the swift and robust normalization following treatment suggest that GBCr may serve as a treatment response biomarker for the development of rapid acting antidepressants. The data also identified unique prefrontal and striatal circuitry as a putative marker of successful treatment and a target for antidepressants' development.

Neuropsychopharmacology (2017) 42, 1210-1219; doi:10.1038/npp.2016.186; published online 12 October 2016
\end{abstract}

\section{INTRODUCTION}

Resting-state functional connectivity magnetic resonance imaging ( $r s-f c \mathrm{MRI})$ is a powerful tool for interrogating in vivo large-scale brain networks in health and disease (Anticevic et al, 2012). Major depressive disorder (MDD), a debilitating mental illness with high rates of treatment nonresponse, shows widespread dysconnectivity as measured by $r s-f c$ MRI (Kaiser et al, 2015). Early studies have primarily used seed-based methods to identify altered networks in MDD (Kaiser et al, 2015). Such approaches are limited by the need for a priori selection of a seed area, and novel analyses

*Correspondence: Dr JW Murrough, Mood and Anxiety Disorders Program, Department of Psychiatry, Icahn School of Medicine at Mount Sinai, One Gustave L. Levy Place, Box 1230, New York, NY I0029, USA, Tel: +I 212 24l 7574, Fax: +I 212 24l 3354, E-mail: james.murrough@mssm.edu

Received 31 May 2016 ; revised 26 July 20 16; accepted 22 August 2016; accepted article preview online 8 September 2016 have been developed to conduct fully data-driven assessment of whole-brain connectivity without the need for seed selection (Bullmore and Sporns, 2009; Menon, 2011). Here we used a recently implemented and optimized graph-based measure, termed global brain connectivity with global signal regression (GBCr), to quantify functional dysconnectivity measured via resting-state blood-oxygen-level-dependent (BOLD) fMRI in MDD patients (Cole et al, 2011). We examined $\mathrm{GBCr}$ at baseline during a depressive episode and $24 \mathrm{~h}$ following administration of a novel putative rapidly acting antidepressant, ketamine.

Ketamine, an $N$-methyl- $D$-aspartate receptor (NMDAR) antagonist, exerts rapid antidepressant effects with a response rate of $50-60 \%$ in treatment-resistant MDD patients. The peak response rates have been reported within $24 \mathrm{~h}$ after administration of a single subanesthetic dose (typically $0.5 \mathrm{mg} / \mathrm{kg}$ ) (Murrough et al, 2013; Zarate et al, 2006). This rapid mood effect, even in chronic and severely depressed patients, offers a unique pharmacoimaging paradigm to investigate the neural correlates of antidepressant 
response as well as to determine state $v s$ trait brain abnormalities in MDD. This pharmacological paradigm also allows the investigation of the neurobiological mechanisms hypothesized to underlie depression and antidepressants' effect. Preclinical models of depression and chronic stress show prefrontal synaptic dysconnectivity and homeostasis as the underlying mechanism of depression and rapid acting antidepressants, respectively (Abdallah et al, 2015). These studies demonstrate reduced brain neurotrophins, dendritic arborization, spine density, and synaptic strength following chronic stress, which rapidly normalize within $24 \mathrm{~h}$ of ketamine treatment (Duman and Aghajanian, 2012). Consistent with this model, central (eg reduced hippocampal volume) and peripheral abnormalities (eg, reduced brainderived neurotrophic factor (BDNF)) have been demonstrated in depression and other stress-related psychiatric disorders (MacQueen and Frodl, 2011; Sen et al, 2008). In addition, synaptic strength enhancement following ketamine treatment was recently reported in humans and was related to increased $\mathrm{BDNF}$ and treatment response in depressed patients (Duncan et al, 2013). It remains unknown, however, if the rapid enhancement of synaptic structure and function observed in animal models following ketamine can be demonstrated in humans at the level of functional connectivity in patients with depression.

Studies in depression have reported functional connectivity abnormalities within the prefrontal cortex (PFC) and other cortical and subcortical regions. However, owing to the significant heterogeneity of approaches and findings, the essential network alterations in MDD have yet to be determined (Kaiser et al, 2015). In an effort to implement a simple, robust biomarker of intrinsic brain networks and to circumvent some of the seed-based analysis limitations, here we used GBCr to quantify brain dysconnectivity in MDD and to relate these abnormalities to rapid mood normalization following treatment in a fully data-driven manner. Accumulating evidence indicates that $\mathrm{GBCr}$ is a valid biomarker of large-scale intrinsic brain networks. $\mathrm{GBCr}$ correlates with normal brain functions (eg, cognition) (Cole et al, 2012) and with regional cerebral blood flow (rCBF) (Liang et al, 2013). Moreover, GBCr has been successfully used to identify alterations across major networks in clinical conditions. Reduced PFC GBCr was reported in bipolar, chronic schizophrenia, and obsessive-compulsive disorders; all of which harbor a strong component of chronic stress and were previously related to glutamate synaptic homeostasis (Anticevic et al, 2013; Anticevic et al, 2015a; Anticevic et al, 2014; Cole et al, 2011). Additionally, ketamine has been repeatedly shown to increase PFC GBCr during ketamine infusion in healthy volunteers (Anticevic et al, 2015a; Driesen et al, 2013a; Driesen et al, 2013b).

Based on the above data, we hypothesize that patients with MDD in a current depressive episode will show GBCr alterations relative to $\mathrm{HC}$, in particular, reduced $\mathrm{PFC}$ GBCr. We hypothesize that the rapid mood normalization following ketamine treatment would parallel a phenomenon of functional connectivity normalization and that the increases in PFC GBCr would be associated with the ketamine antidepressant response.

\section{MATERIALS AND METHODS}

\section{Subjects}

The current study enrolled male and female individuals aged 21-65 years with MDD and healthy control volunteers (HC). Enrollment and treatment procedures have been previously reported (Murrough et al, 2015). Study criteria included negative urine drug screen, no unstable medical illness, and no $\mathrm{MR}$ contraindication. MDD patients had to have failures of two adequate antidepressant trials as determined by a standardized questionnaire, be antidepressants/antipsychotics-free for at least 1 week prior to imaging (as needed benzodiazepines were allowed but withheld the day of each scan), and meet criteria of current major depressive episode with MDD diagnosis as determined by a structured clinical interview. Patients were in a current depressive episode as determined by the SCID-IV and at least moderate severity with score of $\geqslant 32$ on the Inventory of Depressive Symptomatology-Clinician Rated. Current alcohol or substance abuse disorder and lifetime history of bipolar or psychotic disorder were exclusionary. HC were free of lifetime psychiatric illness.

\section{Study Procedures}

Following medical and psychiatric assessment, eligible participants completed baseline structural MRI and $r s$ - $f$ cMRI scans (see Supplementary Figure S1). Within a week of baseline scans, ketamine $0.5 \mathrm{mg} / \mathrm{kg}$ infusion over 40 min was administered intravenously to MDD participants as previously described (Murrough et al, 2015). Briefly, patients were admitted to a clinical research unit; an indwelling catheter was placed in the antecubital vein of the non-dominant arm, and pulse, blood pressure, digital pulse oximetry, and ECG monitoring were instituted. An anesthesiologist administered ketamine hydrochloride via an infusion pump and patients were discharged home following a $4 \mathrm{~h}$ recovery period. MDD patients underwent a repeated $r s-f c M R I$ scan $24 \mathrm{~h}$ after ketamine infusion. Depression severity, at baseline and $24 \mathrm{~h}$ after ketamine, was determined by independent raters blinded to the treatment using the clinician-administered Montgomery-Åsberg Depression Rating Scale (MADRS). Patients were divided into responders and non-responders to treatment. As widely accepted, depression response was defined as $\geqslant 50 \%$ reduction in MADRS scores (Trivedi et al, 2006).

\section{Neuroimaging Data Acquisition}

Philips Achieva 3.0 T X-series MRI using an eight-channel birdcage head coil for radio frequency transmission and reception was used. High-resolution T1-weighted images were collected using a three-dimensional turbo field echo sequence (3D_TFE; repetition time $=7.5 \mathrm{~ms}$; echo time $=3.5 \mathrm{~ms}$; voxel dimensions $=1 \times 1 \times 1 \mathrm{~mm}^{3}$; field of view $=224 \times 224 \mathrm{~mm}^{2}$; flip angle: $8^{\circ}$ ) and 172 sagittal slices. Participants were instructed to rest with their eyes open during the resting-state scan. Resting-state functional data based on the BOLD signal was acquired using a T2*-weighted gradient echo-planar imaging sequence (repetition time $=2000 \mathrm{~ms}$; echo time $=26.6 \mathrm{~ms}$; voxel dimensions $=2.2 \times 2.2 \times 3.25 \mathrm{~mm}^{3}$; field of view $=210 \times$ 
a

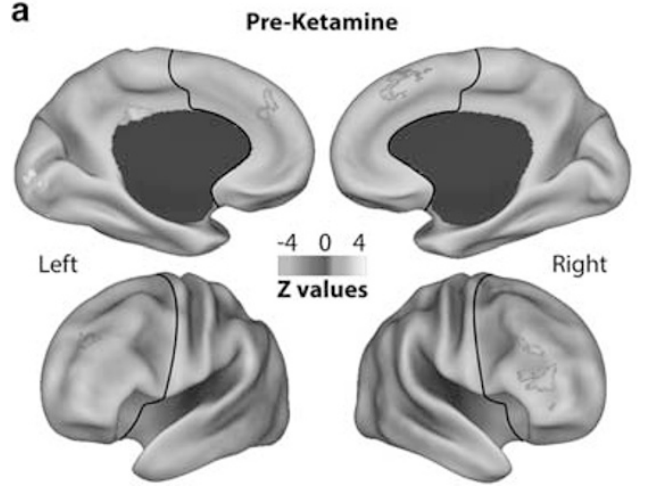

b

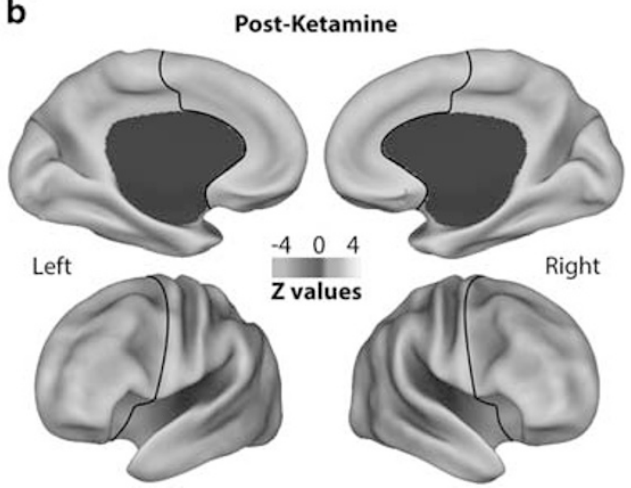

Figure I GBCr alterations in MDD at baseline and following ketamine treatment. Clusters mark brain regions with significant GBCr reduction (blue) or increase (red-yellow) in MDD compared with HC prior to ketamine treatment (a) and $24 \mathrm{~h}$ after intravenous infusion of ketamine (b) (whole-brain voxelwise independent $t$-test with corrected $\alpha<0.05)$. The prefrontal cortex region is labeled with a black line. $\mathrm{HC}$, healthy controls; GBCr, global brain connectivity with global signal regression; MDD, major depressive disorder. A full color version of this figure is available at the Neuropsychopharmacology journal online.

$210 \mathrm{~mm}^{2}$; flip angle $=90^{\circ}$, 120 frames) and 38 contiguous and ascending near-axial slices parallel to the intercommissural (AC-PC). Following the resting-state acquisition, taskbased $f M R I$ and diffusion tensor imaging scans were acquired during the same session. Only the resting-state data were examined in the current report.

\section{GBCr Values}

Details of GBCr methods were previously described (Anticevic et al, 2013; Anticevic et al, 2015a; Anticevic et al, 2014; Anticevic et al, 2015b; Cole et al, 2011; Cole et al, 2012; Driesen et al, 2013a; Driesen et al, 2013b) (see Supplementary Information). GBCr value for each voxel is the average of the correlation between the BOLD time series of a voxel and all other gray matter voxels in the brain. In graph theory terms, Global Brain Connectivity (also known as Functional Connectivity Strength; Liang et al, 2013) is considered a measure of nodal strength of a voxel in the whole brain network-determining brain hubs and examining the coherence between a local region and the rest of the brain (Cole et al, 2010).

\section{Statistical Analyses}

For additional details, see Supplementary Information. Briefly, demographic differences between the study groups were examined using $t$-test and chi-square. Paired $t$-test examined the effect of treatment on depression severity and on GBCr. Independent $t$-tests were conducted to compare voxel-wise GBCr and seed-based maps between groups. Type I error correction was based on peak and cluster extent. All tests are two-tailed with significance set at $p \leqslant 0.05$.

\section{RESULTS}

\section{Clinical Characteristics}

Eighteen MDD and 25 HC participants successfully completed all the study procedures. Age, gender, race, and education did not differ between groups (see Supplementary Table S1). MDD patients on average had a chronic and treatment-refractory course of illness (Supplementary Table
S1). Following ketamine treatment, 10 (56\%) MDD patients achieved response. Paired $t$-test showed a significant effect of treatment on depression severity; delta MADRS $=14.2 \pm 1.9$, $t=7.6, \mathrm{df}=17, p=0.000001$ (Supplementary Figure S2).

\section{GBCr Values Preketamine and Postketamine}

Prior to treatment, whole-brain comparison revealed widespread dysconnectivity in MDD (Figure 1a; Supplementary Figure S3). Altered GBCr voxels were spread over 12 clusters (Table 1). Seven clusters, all of which in the PFC, showed significant reduction in GBCr in MDD compared with HC (Table 1).

Following treatment, GBCr abnormalities of increased or reduced $\mathrm{GBCr}$ in $\mathrm{MDD}$ were divided over three small clusters (Figure 1b; Supplementary Figure S3; Table 1). Examining the changes in GBCr over the treatment period, a paired $t$-test revealed significant increases in the right lateral PFC and reductions in the left cerebellum (Supplementary Figure S4; Table 1).

\section{GBCr and Treatment Response}

Delta GBCr (postketamine-preketamine) was compared using $t$-test between responders and non-responders. Responders showed higher delta GBCr values in the right lateral PFC and the left anterior insula (Supplementary Figure S5). To further assess the robustness of the findings, the results were confirmed by a non-parametric test with 5000 permutations that showed similar clusters (data not shown).

Preketamine showed no GBCr differences between future responders and non-responders. However, postketamine comparison revealed five clusters of significantly higher GBCr in responders compared with non-responders. These clusters were found in the left and right caudate, left and right lateral PFC (IPFC), and left middle temporal (Figure 2). To illustrate, at the individual level, the spread of $\mathrm{GBCr}$ changes in these clusters, we plotted the average delta GBCr (posttreatment - pretreatment) for each of the five clusters in relation to percentage of improvement in MADRS scores (Figure 2). 
Table I GBCr Alteration in MDD at Baseline and $24 \mathrm{~h}$ Following Ketamine Treatment

\begin{tabular}{|c|c|c|c|c|c|c|}
\hline \multicolumn{7}{|l|}{ Preketamine $\mathrm{MDD}<\mathrm{HC}$} \\
\hline Superior medial frontal & $\mathrm{R}$ & $4,24,52$ & 320 & $0.39 \pm 0.07$ & $-0.14 \pm 0.11$ & -1.38 \\
\hline Lateral PFC & $\mathrm{R}$ & $42,44,12$ & 266 & $0.61 \pm 0.06$ & $0.17 \pm 0.07$ & -1.47 \\
\hline Lateral PFC & $\mathrm{L}$ & $-30,42,32$ & 226 & $0.45 \pm 0.08$ & $-0.08 \pm 0.12$ & -1.24 \\
\hline Superior medial frontal & $\mathrm{R}$ & $24,0,60$ & 174 & $0.54 \pm 0.05$ & $0.09 \pm 0.08$ & -1.55 \\
\hline Lateral PFC & $\mathrm{R}$ & $40,28,-4$ & 144 & $0.38 \pm 0.05$ & $-0.02 \pm 0.08$ & -1.49 \\
\hline \multicolumn{7}{|l|}{ Preketamine $M D D>H C$} \\
\hline Lingual & $L$ & $-2,-90,-16$ & 222 & $0.08 \pm 0.08$ & $0.63 \pm 0.09$ & 1.37 \\
\hline \multicolumn{7}{|l|}{ Postketamine $M D D<H C$} \\
\hline Lateral orbitofrontal & $\mathrm{R}$ & $12,16,-22$ & 176 & $-0.01 \pm 0.07$ & $-0.51 \pm 0.09$ & -1.37 \\
\hline \multicolumn{7}{|l|}{ Postketamine MDD $>\mathrm{HC}$} \\
\hline Cerebellum & $L$ & $-28,-78,-22$ & 292 & $-0.38 \pm 0.10$ & $0.30 \pm 0.11$ & 1.42 \\
\hline Cerebellum & $\mathrm{L}$ & $-10,-86,-22$ & 152 & $-0.40 \pm 0.09$ & $0.21 \pm 0.13$ & 1.26 \\
\hline \multicolumn{3}{|c|}{ Postketamine MDD > preketamine MDD } & & Preketamine & Postketamine & \\
\hline
\end{tabular}

Abbreviations: $\mathrm{HC}$, healthy control; L, left; MDD, major depressive disorder; PFC, prefrontal cortex; R, right.

\section{Exploration of GBCr Distributions Preketamine and Postketamine}

To explore the spread of GBCr abnormalities in MDD at baseline and following treatment, we extracted the absolute $z$-values of all brain voxels showing $\mathrm{GBCr}$ alterations preketamine or postketamine (Figure 3a). Preketamine MDD vs HC comparison showed GBCr abnormalities in a total of 2174 voxels (ie, reduced or increased GBCr in MDD with corrected $\alpha<0.05)$. In the postketamine MDD vs HC comparison, 310 voxels differed between groups, of which 123 voxels overlapped with baseline comparison (Figure 3a).

To further delineate the distribution of $\mathrm{GBCr}$ alteration in the PFC, we plotted the average GBCr of each PFC voxel across all subjects in each study group. As shown in Figure 3b, comparison of $\mathrm{PFC} \mathrm{GBCr}$ distributions of preketamine MDD vs HC showed a left shift of large effect size reduction in $\mathrm{PFC} \mathrm{GBCr}$ values in the MDD group,
Cohen's $d=0.95, \mathrm{CI}=(0.91,0.99)$. The PFC GBCr distributions postketamine in responders $v s$ HC largely overlapped with remaining smaller effect size left shift, Cohen's $d=0.46$, $\mathrm{CI}=(0.43,0.50)$ (Figure $3 \mathrm{c}$ ). However, similar to preketamine, we noticed postketamine a large effect size of left shift PFC GBCr distribution in non-responders compared with $\mathrm{HC}$, Cohen's $d=0.92, \mathrm{CI}=(0.87,0.97)$ (Figure $3 \mathrm{~d})$.

\section{Exploratory Seed-Based Analyses}

To provide preliminary data illustrating the circuitry underlying $\mathrm{GBCr}$ alterations, we conducted two follow-up seedbased analyses. The first analysis used data-independent a priori seeds in the affective (subgenual anterior cingulate cortex (sgACC)), cognitive control (dorsolateral PFC (DLPFC)), and default mode networks (posterior cingulate/ precuneus (PCC)). In this analysis, the MDD group showed 

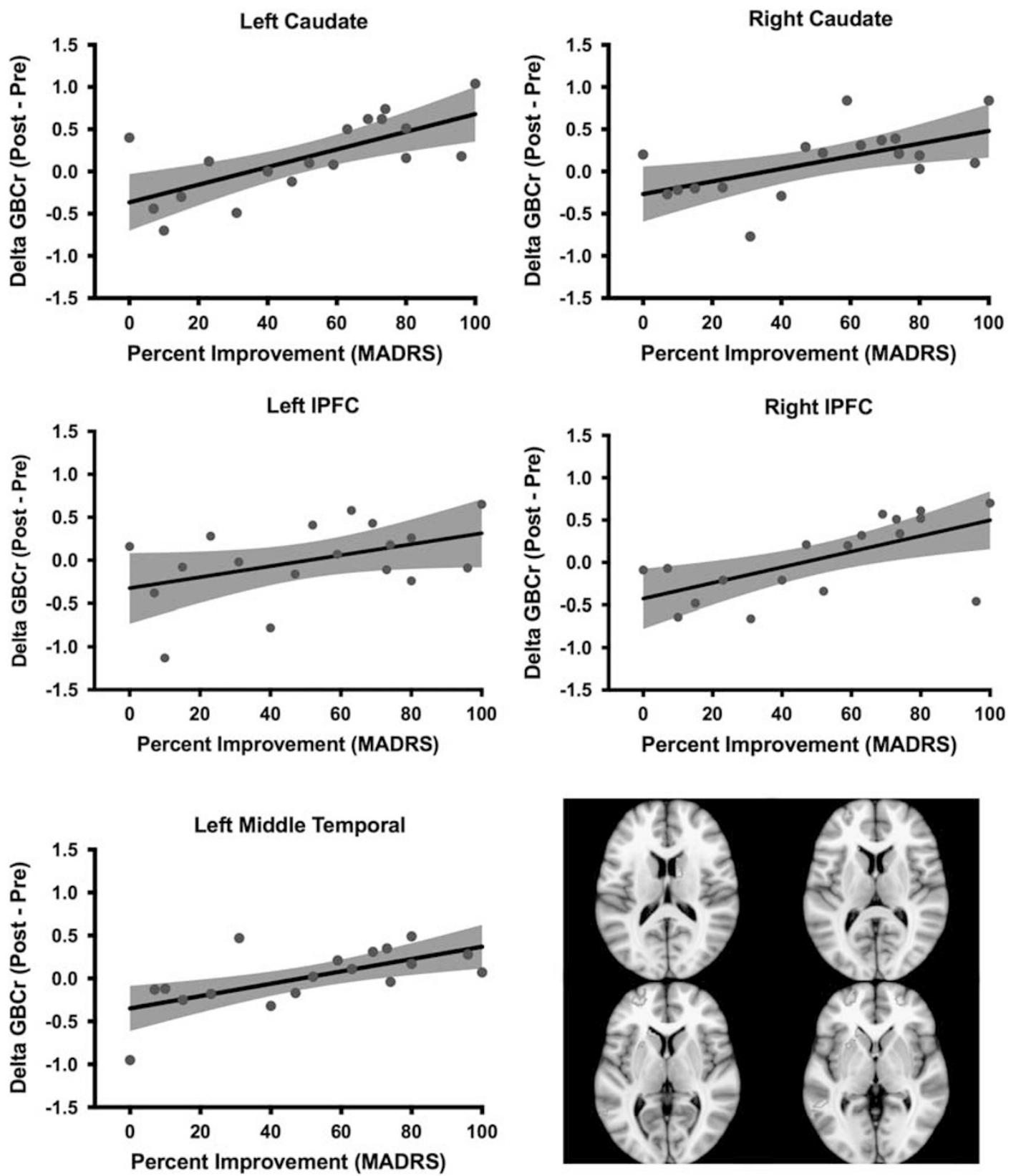

Figure 2 Individual variation in delta GBCr. This figure depict the spread at the subject level of both the percentage of improvement of depressive symptoms and the average GBCr changes in each of the five clusters identified in the responders vs non-responders analysis. The gray area is the $95 \%$ confidence band of the best-fit line. Clusters' locations are shown in right lower panel. GBCr, global brain connectivity with global signal regression; IPFC, lateral prefrontal cortex; MADRS, Montgomery-Åsberg Depression Rating Scale.

an increase in connectivity within the PFC, in contrast with a reduction in connectivity between the PFC and the rest of the brain (Figure $4 \mathrm{a}-\mathrm{c}$ ). Ketamine significantly reduced the connectivity within the PFC and enhanced connectivity between the $\mathrm{PFC}$ and other brain regions (Figure 4d-f).

The second analysis used data-dependent seeds of the four clusters in the IPFC and caudate (Figure 2), which showed increased $\mathrm{GBCr}$ in responders. Compared with non-responders, postketamine seed-based analysis revealed increased IPFC and caudate connectivity with several brain regions outside the PFC/subcortex. However, responders showed lower connectivity within the PFC and subcortical regions (Supplementary Figure S6).

\section{DISCUSSION}

We found reduced GBCr within the PFC in patients with MDD compared with healthy subjects and this largely normalized $24 \mathrm{~h}$ following ketamine. Responders to ketamine treatment evidenced more robust increases in PFC, caudate, and insula GBCr compared with non-responders, implicating enhancement of GBCr in the antidepressant mechanism of action of ketamine. High GBCr values in preketamine MDD 

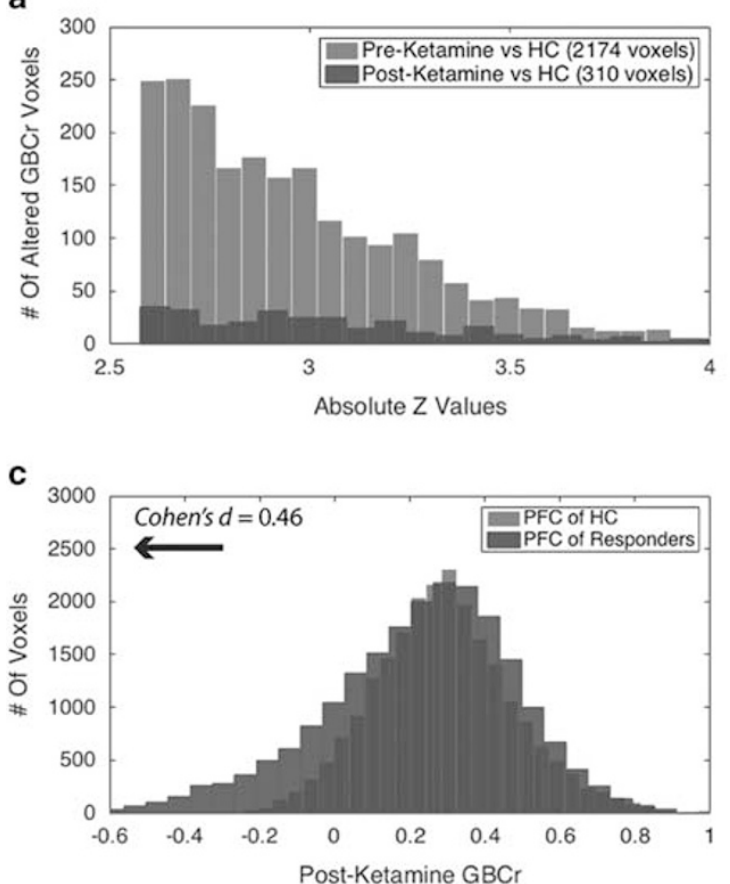

b

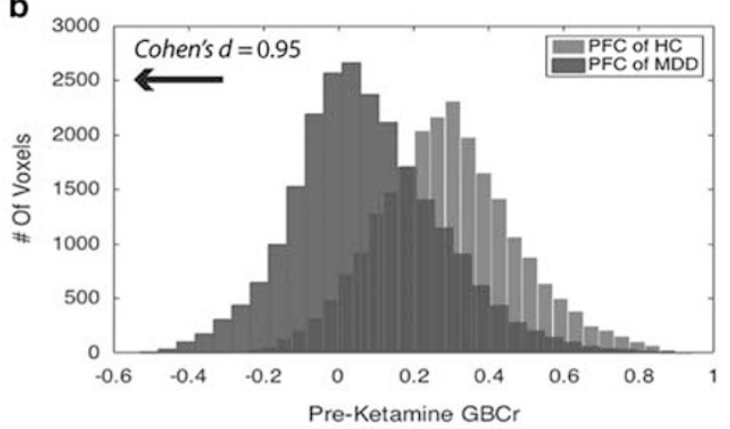

d

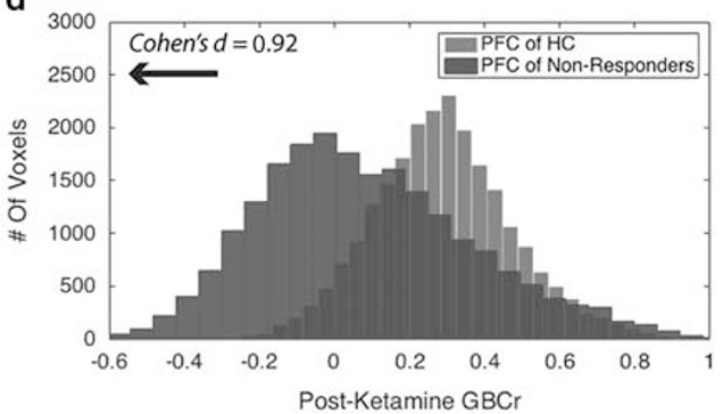

Figure 3 Distributions of $\mathrm{GBCr}$ at baseline and following ketamine treatment. (a) Distributions of absolute z-values of voxels showing significant reduction or increase of $\mathrm{GBCr}$ in MDD compared with $\mathrm{HC}$ prior to ketamine treatment (red) or $24 \mathrm{~h}$ after intravenous infusion of ketamine (blue). Although both distributions largely overlapped, the numbers of altered $\mathrm{GBCr}$ were numerically higher preketamine in each bin of the absolute $z$-values up to $z \sim 3.5$. Of the 310 significant voxels postketamine, 123 were altered at baseline and 187 differed between groups only at the $24 \mathrm{~h}$ time point. (b) Distributions of GBCr in the anatomically defined PFC (delineated in Figure I) in MDD and HC prior to ketamine treatment. The histograms depict comparable normal distributions in both groups with large effect size left shift in MDD reflecting overall reduction in PFC GBCr. (c and d) Distributions of PFC GBCr in HC and MDD responders (c) or MDD non-responders (d) $24 \mathrm{~h}$ after infusion of ketamine. The histograms reveal large overlap between the PFC GBCr distributions of HC and MDD responders (c). However, MDD non-responders continued to show large effect size left shift, suggesting a lack of normalization of PFC GBCr in this subgroup. $\mathrm{GBCr}$, global brain connectivity with global signal regression; HC, healthy controls; MDD, major depressive disorder; PFC, prefrontal cortex. A full color version of this figure is available at the Neuropsychopharmacology journal online.

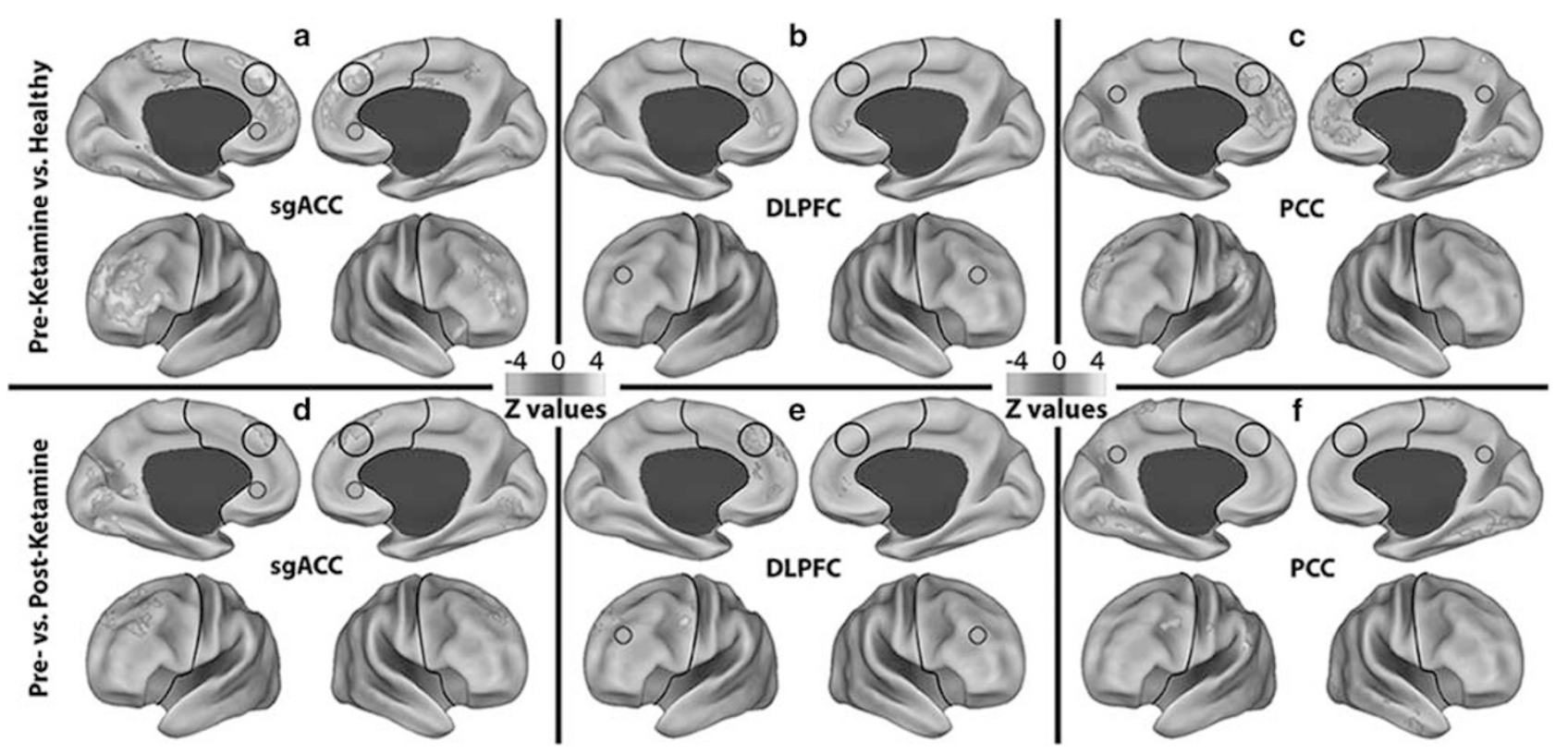

Figure 4 Seed-based connectivity alteration in the affective (AN), cognitive Control (CCN), and default mode networks (DMN). (a-c): Clusters mark brain regions with significant connectivity reduction (blue) or increase (red-yellow) in MDD compared with HC at baseline. (d-f) Clusters mark brain regions with significant connectivity reduction (blue) or increase (red-yellow) in following ketamine treatment. The prefrontal cortex region is labeled with a black line. The seeds' locations are marked with green circles. The empty black circles locate the dorsal nexus area. DLPFC, dorsolateral prefrontal cortex; HC, healthy controls; MDD, major depressive disorder; PCC, posterior cingulate and precuneus area; sgACC, subgenual anterior cingulate cortex. A full color version of this figure is available at the Neuropsychopharmacology journal online. 
compared with $\mathrm{HC}$ were found in the posterior cingulate, precuneus, and occipital cortices; all of which normalized following treatment. Large clusters of high GBCr were also found in the cerebellum, which only partially normalized following treatment. Although baseline GBCr was not related to treatment response, postketamine $\mathrm{GBCr}$ distinguished brain regions, which showed increased GBCr in responders compared with non-responders (Figure 2). Of note, given the very short half-life of ketamine and its active metabolite norketamine (both $<2 \mathrm{~h}$ ), our functional imaging results at $24 \mathrm{~h}$ are not confounded by the presence of ketamine.

A working model of depression and chronic stress proposes prefrontal glutamate synaptic dysconnectivity as a key underlying neural correlate of depression. In this model, antidepressants exert their effects-at least in part-by restoring synaptic homeostasis in the PFC and other brain regions critical to emotion regulation (Abdallah et al, 2015). In the current study, the rapid normalization of functional connectivity following successful treatment with ketamine suggests at least two possibilities. The first interpretation is that the observed GBCr abnormalities are state dependent and are likely to normalize with successful treatment regardless of the specific treatment modality, be it a rapidly acting drug, traditional antidepressant, psychotherapy, or placebo. The second possibility is that GBCr abnormalities are at least partially trait-dependent and related to the etiology of depression; however, the unique mechanisms of ketamine normalized these large-scale abnormalities. These possibilities are not necessarily mutually exclusive, considering the widespread abnormalities and the differential normalization in connectivity across different brain regions. In addition, neurobiological mechanisms of mental disorders such as MDD are likely complex and involve interacting circuitry and molecular alterations (Drevets et al, 2008; Mayberg, 2003). However, concurring with the mathematician George Box that 'all models are wrong but some are useful', we believe that these working hypotheses provide insight into valuable mechanistic questions to be further explored in future studies.

(1) Is GBCr normalization unique to ketamine treatment? A definitive answer could be obtained in future studies including placebo and traditional antidepressant arms to compare with ketamine (see Supplementary Information). Prior evidence suggests that traditional antidepressants may reverse some intrinsic network abnormalities identified using seed-based analyses or other approaches (Wang et al, 2015). However, the swift and robust connectivity networks' normalization revealed in this study were not previously observed.

(2) What is the relationship between GBCr abnormalities and synaptic dysconnectivity? Although speculative in nature, our working hypothesis is that GBCr-under certain circumstances-may reflect overall synaptic strength in a brain region. Considering the tight coupling between synaptic strength, glutamate cycling, and neuroenergetics (Abdallah et al, 2014; Hyder et al, 2013), it is important to highlight that GBCr has been found to positively correlate with rCBF (Liang et al, 2013). Similarly, other GBC-type measures were positively associated with $\mathrm{rCBF}$ and cerebral metabolic rate of glucose (Liang et al, 2014; Tomasi et al, 2013). In this context, the observed PFC reduction in $\mathrm{GBCr}$ could be an indirect measure of chronic stress-induced synaptic dysconnectivity. Consistent with this hypothesis is the rapid normalization, in the current study, of $\mathrm{GBCr}$ following ketamine, which is known to rapidly restore synaptic homeostasis in animal models (Li et al, 2011). Moreover, the GBCr changes were associated with treatment response, which parallel prior evidence showing that the normalization of synaptic connectivity is necessary for successful antidepressant treatments (Duman and Aghajanian, 2012; Gorman and Docherty, 2010).

Prefrontal GBCr reduction has been observed in several chronic psychiatric disorders (ie, bipolar, obsessive-compulsive, and schizophrenia), all of which are influenced by chronic stress (Anticevic et al, 2013; Anticevic et al, 2014; Cole et al, 2011). Acute stress has been found to increase synaptic strength (Yuen et al, 2009; Yuen et al, 2011). In contrast, chronic stress is believed to precipitate glutamatergic dysregulation, leading to increased extracellular glutamate, and excitotoxicity, with subsequent synaptic dysconnectivity and reduced synaptic strength (Bessa et al, 2009; Kang et al, 2012; Yuen et al, 2012). Intriguingly, earlycourse schizophrenia patients were found to have increased PFC GBCr (Anticevic et al, 2015a; Anticevic et al, 2015b). However, following a long course of illness, chronic schizophrenia patients showed significant reduction in PFC GBCr (Anticevic et al, 2015a; Cole et al, 2011). These observations suggest the possibility of increased overall glutamate synaptic strength early in the course of the disorder, comparable to NMDA antagonists' effect-which temporally increase glutamate activities as well as GBCr in healthy volunteers (Anticevic et al, 2015a; Driesen et al, 2013a; Driesen et al, 2013b; Rowland et al, 2005; Stone et al, 2012). However, the chronic glutamate activation-owing to NMDA hypofunction and/or psychopathology-related stress-precipitates PFC synaptic dysconnectivity and GBCr reduction (Krystal and Anticevic, 2015). Together these data support a relationship between GBCr and underlying synaptic strength; however, the circumstances under which this relationship is maintained and the nature of this relationship (causal vs correlational) remain to be ascertained in future studies.

(3) To what extent are the observed GBCr abnormalities specific to $\mathrm{MDD}$, rather than reflecting a nonspecific effect of chronic stress? As described above, it is plausible that GBCr abnormalities are at least partially related to the detrimental effects of chronic stress on synaptic homeostasis. However, this proposed phenomenon is unlikely to explain all the observed GBCr abnormalities in MDD. The interaction between the effects of chronic stress and the unique psychopathology of disorders is likely to lead to differential intrinsic network dysconnectivity, resulting in disease-specific regional abnormalities. For example, although the GBCr alterations were reported in the frontal region of various stress-related psychiatric disorders, there appear to be limited overlap between the locations of abnormal clusters (Anticevic et al, 2013; Anticevic et al, 2015a; Anticevic et al, 2014; Cole et al, 2011). In addition, some regional GBCr abnormalities may be disease specific, eg, putamen in obsessive-compulsive disorder (OCD) (Anticevic et al, 2014). Similar to the current findings in MDD, the increase in cerebellar GBCr was found in OCD (Anticevic et al, 2014). However, the posterior cingulate and precuneus clusters - which may reflect alterations in default 
mode subnetworks-appear to be specific to MDD. It remains to be demonstrated in future study the distribution of GBCr abnormalities in other stress-related disorders, eg, posttraumatic stress disorder (PTSD).

(4) What are the long-term effects of ketamine on GBCr? It is important to highlight that the observed GBCr changes were demonstrated at a single time point and that they occurred following a single infusion of ketamine. Thus it is not known for how long the GBCr changes would last and whether repeated ketamine would have comparable effects. To the extent the observed GBCr changes are related to depression relief and to the ketamine-induced synaptogenesis, we speculate that GBCr changes could last for about 10 days; that is the approximate length of antidepressant response in humans (Bobo et al, 2016) and of the synaptogenic effects in rodents (Duman, 2014). Frequent daily administration of ketamine could lead to repeated glutamate surge, increased extracellular glutamate, excitotoxity, and synaptic dysconnectivity comparable to chronic stress models of depression (Abdallah et al, 2015), consistent with clinical data showing impaired cognition and depressive symptomatology in ketamine abusers (Fan et al, 2016; Morgan et al, 2012). However, much more human research in necessary to determine the long-term safety and efficacy of ketamine, which is not currently FDA approved for depression treatment.

(5) What circuits are putatively involved in successful antidepressant treatment? Supporting previous reports, we found increased sgACC and DLFPC connectivity (which normalized following treatment) with the dorsomedial PFC, an area termed the dorsal nexus and previously implicated in the pathophysiology and treatment of MDD (Scheidegger et al, 2012; Sheline et al, 2010). Integrating the GBCr and seed-based results, we hypothesize that during depression the brain connectivity balance is altered in favor of short distance connectivity within the PFC/subcortex, combined with long distance disconnect with the rest of the brain. Ketamine normalizes this PFC/subcortex dysconnectivity, by enhancing long distance connectivity and restoring the central role of the PFC in global functions. Consistent with this model, the current study identified specific regions in the IPFC and caudate as neural correlates to successful treatment, in which these brain regions showed more central and balanced connectivity in responders. These frontal and striatal regions have a critical role in higher cognitive control, in particular, in exploration and goal-directed behavior (Koechlin et al, 2000; Wang and Voss, 2014). Thus it is plausible that the enhanced engagement of these frontostriatal regions underlies the behavioral shift from depression, withdrawal, and rumination to exploratory and externally focused behavior following recovery.

\section{Limitations}

This study has several limitations. Given the comorbidity of anxiety disorders in the study sample, it is conceivable that anxiety pathology contributed to the GBCr alterations. Also, given the short medication-free period, we cannot rule out the presence of residual effects from prior antidepressant use on GBCr. Although age did not differ statistically between groups $(p=0.88)$, numerically the MDD group was 4 years older than the HC group. The short $r s$-fcMRI scan acquisition is a limitation. Although short resting-state acquisition has previously been shown sufficient (Van Dijk et al, 2010), considering recent findings and advances in addressing motion artifacts, future studies would benefit from longer acquisition time (eg, $3 \times 5 \mathrm{~min}$ sessions) (Birn et al, 2013). Another limitation is the relatively small sample size in the response analysis; thus this finding should be considered preliminary. GBC without global signal regression (GBCnr) failed to detect abnormalities in MDD (see Supplementary Information). It is plausible that GBCrenhanced sensitivity provided increased power to detect differences in the current cohort. It remains to be determined in future larger studies the utility of GBCnr as a biomarker in ketamine-MDD research. Finally, we speculated about the potential mechanisms of GBCr abnormalities and normalization; however, the current report did not per se investigate the underlying mechanisms. Considering the current findings, future studies are encouraged to employ a multimodal approach investigating the structural (eg, diffusion weighted imaging), chemical (eg, synaptic density using SV2A tracer or glutamate cycling using 13C spectroscopy), and functional (eg, task-based fMRI) correlates of GBCr alterations.

The findings of the current report support the use of the GBCr approach to successfully identify intrinsic network dysconnectivity in MDD patients, and provides preliminary evidence regarding the utility of $\mathrm{GBCr}$ as a biomarker of target validation for drug development.

\section{FUNDING AND DISCLOSURE}

This research was supported by the National Institute of Mental Health of the National Institutes of Health under Award Number K23MH094707 (Career Development Award to Dr Murrough). Support was also provided by the Iris \& Junming Le Foundation (Award to Dr Murrough), by the Brain and Behavior Research Foundation (NARSAD awards to Dr Murrough and Dr Abdallah), and by grant UL1TR000067 from the NIH National Center for Advancing Translational Sciences to Mount Sinai. Additional research support was provided by the Patterson Trust Award (Dr Abdallah), the NIHM K23MH101498 (Dr Abdallah), and the US Department of Veterans Affairs via its funding of the VA National Center for PTSD_Clinical Neurosciences Division (Dr Averill and Dr Abdallah) and the Office of Academic Affairs Advanced Fellowship in Mental Illness Research and Treatment (Dr Averill). The content is solely the responsibility of the authors and does not necessarily represent the official views of the National Institutes of Health or other organization. The sponsors had no role in the design and conduct of the study; collection, management, analysis, and interpretation of the data; and preparation, review, or approval of the manuscript. Dr Murrough and Dr Abdallah had full access to all the data in the study and take responsibility for the integrity of the data and the accuracy of the data analysis. In the past 3 years, Dr Murrough has served on advisory boards for Janssen Research and Development and Genentech, has provided consultation services for ProPhase and Impel Neuropharma, and has received research support from Janssen and Avanir Pharmaceuticals; he is named on a patent pending for neuropeptide $\mathrm{Y}$ as a treatment for mood and anxiety disorders and on 
a patent pending for the combination of ketamine and lithium for suicidal ideation. He is an employee of the Icahn School of Medicine at Mount Sinai and has received external funding from the National Institutes of Health, the Brain and Behavioral Research Foundation, and the American Foundation for Suicide Prevention. Dr Abdallah has served on advisory boards for Genentech. He is an employee of the Yale School of Medicine and the VA National Center for PTSD. He has received funding from the National Institutes of Health, the Brain and Behavioral Research Foundation, the Department of Defense, the American Psychiatric Foundation, and the Robert E. Leet and Clara Guthrie Patterson Trust. Dr Iosifescu has consulted for Avanir, CNS Response, INSYS Therapeutics, Lundbeck, Otsuka, Servier, and Sunovion and he has received grant/research support through Mount Sinai School of Medicine from Alkermes, Astra Zeneca, Brainsway, Euthymics Bioscience Inc, Neosync, Roche, and Shire. Dr Dennis Charney (Dean of Icahn School of Medicine at Mount Sinai), and Icahn School of Medicine at Mount Sinai have been named on a use patent on ketamine for the treatment of depression. Dr Charney and Icahn School of Medicine at Mount Sinai could potentially benefit if ketamine were to gain approval for the treatment of depression. Dr Charney is named on a patent pending for ketamine as a treatment for PTSD and for neuropeptide $\mathrm{Y}$ as a treatment for mood and anxiety disorders; he has received funding from the US Department of Defense, NIH, NIH/NIMH, NARSAD, and USAMRAA; he has served on the scientific advisory board for the Institute of Medicine Committee on DHS Workforce Resilience and on the editorial board of CNS Spectrums. The other authors declare no conflict of interest.

\section{ACKNOWLEDGMENTS}

We thank all the study participants for their invaluable contribution.

\section{REFERENCES}

Abdallah CG, Jiang L, De Feyter HM, Fasula M, Krystal JH, Rothman DL et al (2014). Glutamate metabolism in major depressive disorder. Am J Psychiatry 171: 1320-1327.

Abdallah CG, Sanacora G, Duman RS, Krystal JH (2015). Ketamine and rapid-acting antidepressants: a window into a new neurobiology for mood disorder therapeutics. Annu Rev Med 66: 509-523.

Anticevic A, Brumbaugh MS, Winkler AM, Lombardo LE, Barrett J, Corlett PR et al (2013). Global prefrontal and fronto-amygdala dysconnectivity in bipolar I disorder with psychosis history. Biol Psychiatry 73: 565-573.

Anticevic A, Cole MW, Murray JD, Corlett PR, Wang XJ, Krystal JH (2012). The role of default network deactivation in cognition and disease. Trends Cogn Sci 16: 584-592.

Anticevic A, Corlett PR, Cole MW, Savic A, Gancsos M, Tang Y et al (2015a). N-methyl-D-aspartate receptor antagonist effects on prefrontal cortical connectivity better model early than chronic schizophrenia. Biol Psychiatry 77: 569-580.

Anticevic A, Hu S, Zhang S, Savic A, Billingslea E, Wasylink S et al (2014). Global resting-state functional magnetic resonance imaging analysis identifies frontal cortex, striatal, and cerebellar dysconnectivity in obsessive-compulsive disorder. Biol Psychiatry 75: 595-605.
Anticevic A, Hu X, Xiao Y, Hu J, Li F, Bi F et al (2015b). Early-course unmedicated schizophrenia patients exhibit elevated prefrontal connectivity associated with longitudinal change. J Neurosci 35: 267-286.

Bessa JM, Ferreira D, Melo I, Marques F, Cerqueira JJ, Palha JA et al (2009). The mood-improving actions of antidepressants do not depend on neurogenesis but are associated with neuronal remodeling. Mol Psychiatry 14: 764-773 739.

Birn RM, Molloy EK, Patriat R, Parker T, Meier TB, Kirk GR et al (2013). The effect of scan length on the reliability of resting-state fMRI connectivity estimates. Neuroimage 83: 550-558.

Bobo WV, Voort JL, Croarkin PE, Leung JG, Tye SJ, Frye MA (2016). Ketamine for treatment-resistant unipolar and bipolar major depression: critical review and implications for clinical practice. Depress Anxiety 33: 698-710.

Bullmore E, Sporns O (2009). Complex brain networks: graph theoretical analysis of structural and functional systems. Nat Rev Neurosci 10: 186-198.

Cole MW, Anticevic A, Repovs G, Barch D (2011). Variable global dysconnectivity and individual differences in schizophrenia. Biol Psychiatry 70: 43-50.

Cole MW, Pathak S, Schneider W (2010). Identifying the brain's most globally connected regions. Neuroimage 49: 3132-3148.

Cole MW, Yarkoni T, Repovs G, Anticevic A, Braver TS (2012). Global connectivity of prefrontal cortex predicts cognitive control and intelligence. J Neurosci 32: 8988-8999.

Drevets WC, Price JL, Furey ML (2008). Brain structural and functional abnormalities in mood disorders: implications for neurocircuitry models of depression. Brain Struct Funct 213: 93-118.

Driesen NR, McCarthy G, Bhagwagar Z, Bloch M, Calhoun V, D'Souza DC et al (2013a). Relationship of resting brain hyperconnectivity and schizophrenia-like symptoms produced by the NMDA receptor antagonist ketamine in humans. Mol Psychiatry 18: 1199-1204.

Driesen NR, McCarthy G, Bhagwagar Z, Bloch MH, Calhoun VD, D'Souza DC et al (2013b). The impact of NMDA receptor blockade on human working memory-related prefrontal function and connectivity. Neuropsychopharmacology 38: 2613-2622.

Duman RS (2014). Pathophysiology of depression and innovative treatments: remodeling glutamatergic synaptic connections. Dialogues Clin Neurosci 16: 11-27.

Duman RS, Aghajanian GK (2012). Synaptic dysfunction in depression: potential therapeutic targets. Science 338: 68-72.

Duncan WC, Sarasso S, Ferrarelli F, Selter J, Riedner BA, Hejazi NS et al (2013). Concomitant BDNF and sleep slow wave changes indicate ketamine-induced plasticity in major depressive disorder. Int J Neuropsychopharmacol 16: 301-311.

Fan N, Xu K, Ning Y, Rosenheck R, Wang D, Ke X et al (2016). Profiling the psychotic, depressive and anxiety symptoms in chronic ketamine users. Psychiatry Res 237: 311-315.

Gorman JM, Docherty JP (2010). A hypothesized role for dendritic remodeling in the etiology of mood and anxiety disorders. J Neuropsychiatry Clin Neurosci 22: 256-264.

Hyder F, Rothman DL, Bennett MR (2013). Cortical energy demands of signaling and nonsignaling components in brain are conserved across mammalian species and activity levels. Proc Natl Acad Sci USA 110: 3549-3554.

Kaiser RH, Andrews-Hanna JR, Wager TD, Pizzagalli DA (2015). Large-scale network dysfunction in major depressive disorder: a meta-analysis of resting-state functional connectivity. JAMA Psychiatry 72: 603-611.

Kang HJ, Voleti B, Hajszan T, Rajkowska G, Stockmeier CA, Licznerski P et al (2012). Decreased expression of synapse-related genes and loss of synapses in major depressive disorder. Nat Med 18: 1413-1417.

Koechlin E, Corrado G, Pietrini P, Grafman J (2000). Dissociating the role of the medial and lateral anterior prefrontal cortex in human planning. Proc Natl Acad Sci USA 97: 7651-7656. 
Krystal JH, Anticevic A (2015). Toward illness phase-specific pharmacotherapy for schizophrenia. Biol Psychiatry 78: 738-740.

Li N, Liu RJ, Dwyer JM, Banasr M, Lee B, Son H et al (2011). Glutamate N-methyl-D-aspartate receptor antagonists rapidly reverse behavioral and synaptic deficits caused by chronic stress exposure. Biol Psychiatry 69: 754-761.

Liang X, Connelly A, Calamante F (2014). Graph analysis of restingstate ASL perfusion MRI data: nonlinear correlations among CBF and network metrics. Neuroimage 87: 265-275.

Liang X, Zou Q, He Y, Yang Y (2013). Coupling of functional connectivity and regional cerebral blood flow reveals a physiological basis for network hubs of the human brain. Proc Natl Acad Sci USA 110: 1929-1934.

MacQueen G, Frodl T (2011). The hippocampus in major depression: evidence for the convergence of the bench and bedside in psychiatric research? Mol Psychiatry 16: 252-264.

Mayberg HS (2003). Modulating dysfunctional limbic-cortical circuits in depression: towards development of brain-based algorithms for diagnosis and optimised treatment. Br Med Bull 65: 193-207.

Menon V (2011). Large-scale brain networks and psychopathology: a unifying triple network model. Trends Cogn Sci 15: 483-506.

Morgan CJ, Curran HVIndependent Scientific Committee on Drugs (2012). Ketamine use: a review. Addiction 107: 27-38.

Murrough JW, Collins KA, Fields J, DeWilde KE, Phillips ML, Mathew SJ et al (2015). Regulation of neural responses to emotion perception by ketamine in individuals with treatment-resistant major depressive disorder. Transl Psychiatry 5: e509.

Murrough JW, Iosifescu DV, Chang LC, Al Jurdi RK, Green CE, Perez AM et al (2013). Antidepressant efficacy of ketamine in treatment-resistant major depression: a two-site randomized controlled trial. Am J Psychiatry 170: 1134-1142.

Rowland LM, Bustillo JR, Mullins PG, Jung RE, Lenroot R, Landgraf $\mathrm{E}$ et al (2005). Effects of ketamine on anterior cingulate glutamate metabolism in healthy humans: a 4 -T proton MRS study. Am J Psychiatry 162: 394-396.

Scheidegger M, Walter M, Lehmann M, Metzger C, Grimm S, Boeker $\mathrm{H}$ et al (2012). Ketamine decreases resting state functional network connectivity in healthy subjects: implications for antidepressant drug action. PLoS One 7: e44799.

Sen S, Duman R, Sanacora G (2008). Serum brain-derived neurotrophic factor, depression, and antidepressant medications: meta-analyses and implications. Biol Psychiatry 64: 527-532.
Sheline YI, Price JL, Yan Z, Mintun MA (2010). Resting-state functional MRI in depression unmasks increased connectivity between networks via the dorsal nexus. Proc Natl Acad Sci USA 107: 11020-11025.

Stone JM, Dietrich C, Edden R, Mehta MA, De Simoni S, Reed LJ et al (2012). Ketamine effects on brain GABA and glutamate levels with $1 \mathrm{H}$-MRS: relationship to ketamine-induced psychopathology. Mol Psychiatry 17: 664-665.

Tomasi D, Wang GJ, Volkow ND (2013). Energetic cost of brain functional connectivity. Proc Natl Acad Sci USA 110: 13642-13647.

Trivedi MH, Rush AJ, Wisniewski SR, Nierenberg AA, Warden D, Ritz L et al (2006). Evaluation of outcomes with citalopram for depression using measurement-based care in $\mathrm{STAR}^{\star} \mathrm{D}$ : implications for clinical practice. Am J Psychiatry 163: $28-40$.

Van Dijk KR, Hedden T, Venkataraman A, Evans KC, Lazar SW, Buckner RL (2010). Intrinsic functional connectivity as a tool for human connectomics: theory, properties, and optimization. J Neurophysiol 103: 297-321.

Wang JX, Voss JL (2014). Brain networks for exploration decisions utilizing distinct modeled information types during contextual learning. Neuron 82: 1171-1182.

Wang L, Xia M, Li K, Zeng Y, Su Y, Dai W et al (2015). The effects of antidepressant treatment on resting-state functional brain networks in patients with major depressive disorder. Hum Brain Mapp 36: 768-778.

Yuen EY, Liu W, Karatsoreos IN, Feng J, McEwen BS, Yan Z (2009). Acute stress enhances glutamatergic transmission in prefrontal cortex and facilitates working memory. Proc Natl Acad Sci USA 106: 14075-14079.

Yuen EY, Liu W, Karatsoreos IN, Ren Y, Feng J, McEwen BS et al (2011). Mechanisms for acute stress-induced enhancement of glutamatergic transmission and working memory. Mol Psychiatry 16: $156-170$.

Yuen EY, Wei J, Liu W, Zhong P, Li X, Yan Z (2012). Repeated stress causes cognitive impairment by suppressing glutamate receptor expression and function in prefrontal cortex. Neuron 73: 962-977.

Zarate CA Jr., Singh JB, Carlson PJ, Brutsche NE, Ameli R, Luckenbaugh DA et al (2006). A randomized trial of an $\mathrm{N}$-methyl-D-aspartate antagonist in treatment-resistant major depression. Arch Gen Psychiatry 63: 856-864.

Supplementary Information accompanies the paper on the Neuropsychopharmacology website (http://www.nature.com/npp) 